\title{
Development of the piezoelectric motor using momentum generated by bimorphs
}

\author{
Hyun Do Choi, ${ }^{a)}$ Jun Hyung Kim, Soohyun Kim, and Yoon Keun Kwak \\ Department of Mechanical Engineering, Korea Advanced Institute of Science and Technology, \\ 373-1, Guseong-dong, Yuseong-gu, Daejeon 305-701, Republic of Korea
}

(Received 12 July 2005; accepted 1 September 2005; published online 25 October 2005)

\begin{abstract}
Piezoelectric motors have been used in many applications where excellent controllability and fine position resolution are required and/or magnetic-field noise should be eliminated. However, the piezoelectric motor has two major drawbacks, difficulty in maintaining vibration amplitude constantly with temperature rise and wear and heat generation induced by dielectric and mechanical losses. In this article, a new piezoelectric motor that can overcome these problems is proposed. The proposed piezoelectric motor is operated using momentum exchange between four bimorphs and a rotor. In order to maximize the steady-state velocity and static torque of the proposed motor, a guideline is established using two bimorph models. The guideline is partially verified by comparison between simulations and experiments. There was no heat generation in a few hours of operation in experiments. (C) 2005 American Institute of Physics. [DOI: 10.1063/1.2084427]
\end{abstract}

\section{INTRODUCTION}

There is currently a demand in various areas such as medical instruments, micromachining, and the electronics industry for a miniaturized motor that can generate high torque or fine resolution. Though different specifications are required in many applications, the important parameters when the motor is miniaturized are the efficiency of the motor and the design of the gear box. To date, electromagnetic motors have been utilized to meet these demands. While these motors still dominate the industry, drastic improvement cannot be expected except through new discoveries in magnetic or superconducting materials. Regarding conventional electromagnetic motors, tiny motors smaller than $1 \mathrm{~cm}^{3}$ are rather difficult to produce with sufficient energy efficiency and they are placed at a disadvantageous position in terms of miniaturization. ${ }^{1}$

An alternative to electrostatics is piezoelectricity. Although piezoelectric motors have various advantages such as rapid response time, ability of direct drive, high position resolution, and simple structure, in the past the motors were not of much practical use because of the difficulty in maintaining a constant vibration amplitude with temperature rise, wear, heat generation, and tear. ${ }^{2-6}$

This article proposes a new piezoelectric motor that can overcome excessive heat generation and wear induced by friction. The proposed motor is operated by momentum exchange between bimorphs and a rotor.

\section{DESIGN OF PIEZOELECTRIC MOTOR}

Figure 1 shows an overview of the developed motor. The rotor is basically disk shaped with an added shaft. ac voltage is applied to the four bimorphs attached to the inclined side of the stator so as to vibrate in resonant frequency.

The driving principle of the motor is as follows. Large momentum is generated to rotate the rotor by the bimorphs, creating a large deflection relative to stacked piezoelectric materials. Using this momentum, rotary motion is induced as the vibrating ends of the bimorphs impact the edge of the rotor. In other words, the motor is operated by a momentum exchange induced by vibration of the bimorphs.

Based on this principle, we reasoned that the proposed motor can overcome some problems that are unavoidable in previous piezoelectric motors, because the impact results in less wear and heat generation than that induced by friction. Furthermore, as the length of bimorph decreases with constant thickness, the blocked force, which means the force measured when the deflection is zero, increases. This feature could allow the motor to maintain high efficiency and torque even if the motor is fabricated in a few millimeter scale.

In order to estimate the characteristics of the steady-state velocity and torque of the proposed motor, modeling is car-

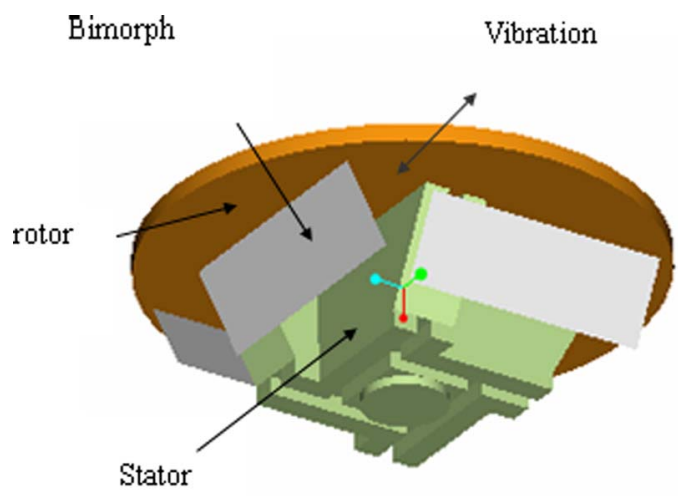

FIG. 1. Overview of the proposed piezoelectric motor.

\footnotetext{
${ }^{\text {a) }}$ Author to whom correspondence should be addressed; electronic mail: chlguseh80@kaist.ac.kr
} 


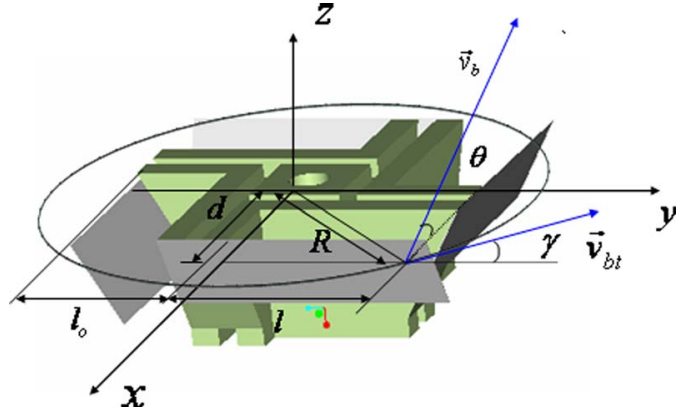

FIG. 2. Velocity of bimorph's end.

ried out. As illustrated in Fig. 2, $\boldsymbol{\nu}_{b}$ is a vector describing the velocity of the bimorph's end and $\boldsymbol{\nu}_{\mathrm{bt}}$ is a vector describing a tangential component of $\boldsymbol{\nu}_{b}$ to the outer circle of the rotor. We also predicted the parameters that will have a dominant effect on the maximum angular velocity and static torque of the proposed motor, which are, in general, key performance indices of the motor.

To establish a design guideline, it is assumed that the angular velocity is proportional to the tangential velocity of the bimorph's end and the static torque is proportional to a tangential component of the momentum generated by the bimorph. This assumption is reasonable considering that the rotation of the rotor is induced by impact and large momentum can be converted to high force with finite time.

From Fig. 2, Eqs. (1) and (2) can be derived with a geometric relation. These are performance indices that indicate the qualitative characteristic of velocity and torque.

$$
\begin{aligned}
& f_{\nu}=\left|\boldsymbol{\nu}_{\mathbf{b t}}\right|_{\max }=\delta \omega \sin \gamma \cos \theta, \\
& f_{m}=m_{\mathrm{eff}}\left|\boldsymbol{\nu}_{\mathrm{bt}}\right|_{\text {max }}=m_{\mathrm{eff}} \delta \omega \sin \gamma \cos \theta
\end{aligned}
$$

where $\delta, \omega$, and $m_{\text {eff }}$ are the deflection, driving frequency, and effective mass. $\gamma$ is calculated from geometry, as given by Eq. (3).

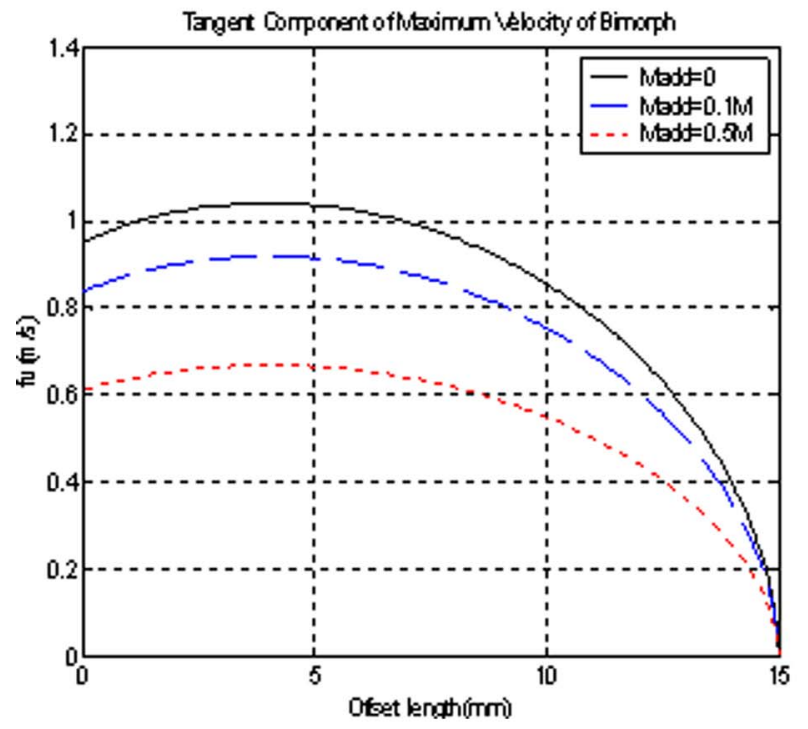

(a)

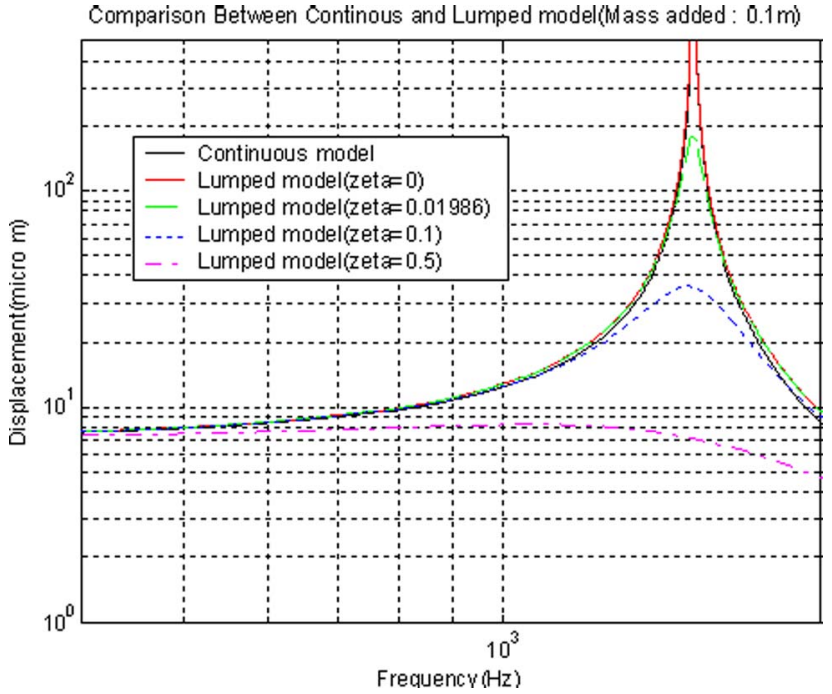

FIG. 3. Comparison between continuous model and lumped model $\left(m_{\text {eff }}=0.355 \rho A l\right)$.

$\sin \gamma=\frac{\sqrt{R^{2}-d^{2}}}{R}$.

Modeling of the bimorph is required to determine the effects of $\delta, \omega$, and $m_{\text {eff }}$ on $f_{\nu}$ and $f_{m}$.

When the bimorph is driven by ac voltage, the voltage across the piezoelectric elements causes one of these elements to contract and the other to expand, and this bends the bimorph periodically. This raises an important question: "How do we represent the internal force that bends the bimorph?" Smits and Ballato answered this question by noting that the forced strain in each of the elements is equal to $\pm d_{31} E_{3}$, where $E_{3}$ is the electric field in each of the elements. This means that if, for instance, we restrain the bimorph from bending by an externally applied moment, the forced stress is also uniform throughout both elements, and equal to $\pm d_{31} E_{3} / s_{11}^{E}$. In conclusion, we have a uniform and internally

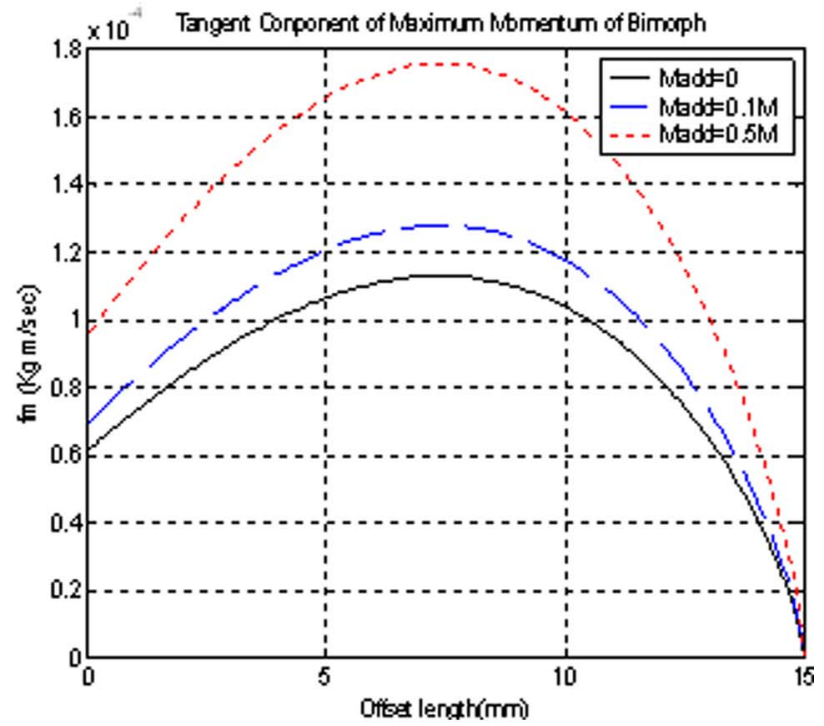

(b)

FIG. 4. Effect of offset distance and added mass on $f_{\nu}$ and $f_{m}$. (a) $f_{\nu}$ vs added mass and offset distance. (b) $f_{m}$ vs added mass and offset distance. 


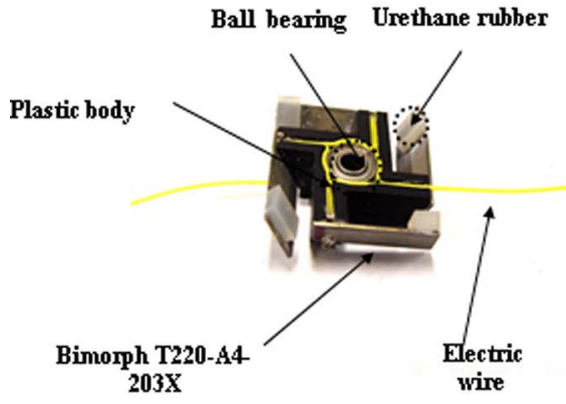

(a)

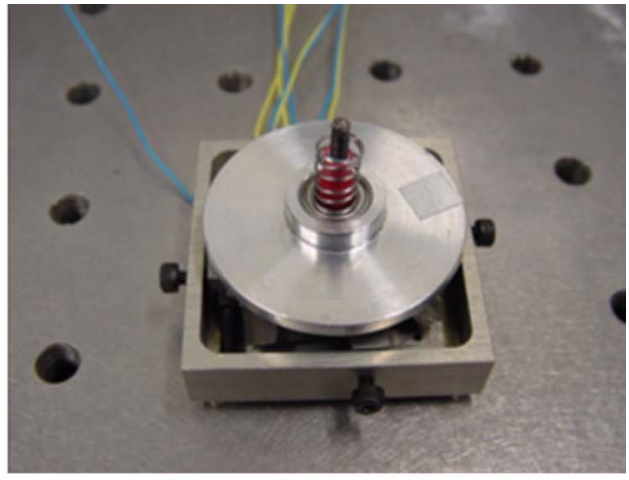

(b)
FIG. 5. Overview of the proposed piezoelectric motor. (a) Components. (b) Fabricated motor. generated moment inside the bimorph, and we now bring that outside as an externally applied moment, as this would constitute a uniform internal moment. ${ }^{7}$ The total moment in the bimorph is

$$
M=2 \int_{0}^{h / 2} z \frac{d_{31} E_{3}}{s_{11}^{E}} w d z=\frac{d_{31} V w h}{4 s_{11}^{E}} .
$$

To derive the governing equation and boundary condition, we applied the extended Hamilton's equation to the bimorph, which has added mass in the tip.

$$
\begin{aligned}
& m \frac{\partial^{2} w}{\partial t^{2}}+\frac{\partial^{2}}{\partial x^{2}}\left(E I \frac{\partial^{2} w}{\partial x^{2}}\right)=0, \\
& E I \frac{\partial^{2} w}{\partial x^{2}}-M \sin \Omega t=0, \quad x=l,
\end{aligned}
$$

$$
-m_{\text {add }} \frac{\partial^{2} w}{\partial t^{2}}+\frac{\partial}{\partial x}\left(E I \frac{\partial^{2} w}{\partial x^{2}}\right)=0, \quad x=l,
$$

$$
\frac{\partial w}{\partial x}=0, \quad x=0, \quad w=0, \quad x=0 .
$$

From the expansion theorem, the beam displacement response can be assumed as given by Eqs. (7) and (8). Here, we can calculate the steady-state response that satisfies the boundary conditions. ${ }^{7,8}$

$$
w(x, t)=\sum_{n=0}^{\infty} W_{n}(w) q_{n}(t)=W(x) q(t),
$$

where $l, E$, and $\Omega$ are the bimorph length, Young's modulus, and the operation frequency.

While we can estimate the deflection of the tip, which is a function of dimension, voltage, piezoelectric materials, and frequency, the derived response has an infinite deflection in the resonant frequency, which cannot exist in reality. Thus, the dissipation of internal energy should be taken into account in order to predict the velocity and torque of the proposed motor correctly.

To take the energy dissipation into consideration, we established a simple one degree of freedom (DOF) model based on the aspect that the bimorph is operated in first reso- nance and the energy dissipation can be considered as a damper. The deflection curve with moment $M$ applied at the tip of the bimorph is described in Eq. (9).

$$
\delta(x)=\frac{M}{2 E I} x^{2} .
$$

The effective stiffness can be derived from Eq. (11), and corresponds with the equivalence of potential energy between the continuous model and the simple one DOF model.

$$
V(t)_{\max }=\frac{1}{2} \int_{0}^{l}\left[E I\left(\frac{\partial^{2} w}{\partial x_{\max }^{2}}\right)^{2}\right] d x=\frac{1}{2}\left(\frac{4 E I}{l^{3}}\right) \delta(l)^{2},
$$




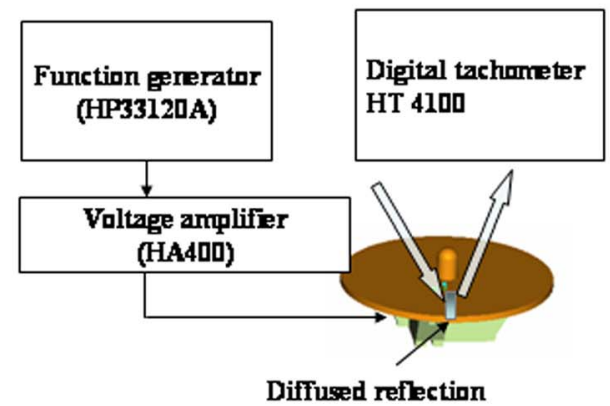

(a)

$$
K_{\text {eff }}=\frac{4 E I}{l^{3}} \text {. }
$$

The effective mass can be derived from Eq. (12), and corresponds with the equivalence of kinetic energy.

$$
\begin{aligned}
& T(t)_{\max }=\frac{1}{2} \int_{0}^{l}\left(\frac{\partial w}{\partial t_{\max }}\right)^{2} \rho A d x=\frac{1}{2}\left(\frac{\rho A l}{5}\right) \delta(l)^{2} \omega^{2}, \\
& m_{\mathrm{eff}}=\frac{\rho A l}{5} .
\end{aligned}
$$

Finally, based on the deflection of the bimorph tip, the effective force can be calculated as given by Eq. (14).

$$
\begin{aligned}
& \delta(l)=\frac{l^{2} M}{2 E I}=\frac{1}{k_{\text {eff }}}\left(\frac{2 M}{l}\right), \\
& F_{\text {eff }}=\frac{2 M}{l} .
\end{aligned}
$$

If we consider the energy dissipation as a damping coefficient, which is obtained from the $Q$ factor in the experiment, the maximum deflection is derived as

$$
\delta(l)=\frac{F_{\mathrm{eff}}}{\sqrt{\left(k_{\mathrm{eff}}-m_{\mathrm{eff}} \omega^{2}\right)^{2}+c^{2} \omega^{2}}} .
$$

Figure 3 shows the frequency responses of the continuous model and the one DOF model, of which the effective mass is adjusted to describe the deflection correctly. From the one DOF model, we confirmed that the resonant frequency and amplitude can be estimated accurately in comparison with the experimentally obtained values.

As given by Eqs. (17) and (18), the performance indices can be established by substituting Eq. (16) into Eqs. (1) and (2).

$$
f_{\nu}=\left|\boldsymbol{\nu}_{\mathrm{bt}}\right|_{\max }=\frac{F_{\mathrm{eff}}}{\sqrt{\left(k_{\mathrm{eff}}-m_{\mathrm{eff}} \omega^{2}\right)^{2}+c^{2} \omega^{2}}} \omega \frac{\sqrt{R^{2}-d^{2}}}{R} \cos \theta,
$$

TABLE I. Parameters of fabricated piezoelectric motor

\begin{tabular}{lrrr}
\hline \hline & Type1 & Type2 & Type3 \\
\hline Diameter of rotor (mm) & 30 & 30 & 30 \\
Offset length of bimorph (mm) & 10 & 9 & 6 \\
Inclined angle of bimorph & $30^{\circ}$ & $20^{\circ}$ & $20^{\circ}$ \\
\hline \hline
\end{tabular}

$$
\begin{aligned}
f_{m} & =m_{\text {eff }}\left|\boldsymbol{\nu}_{\mathbf{b t}}\right|_{\max } \\
& =m_{\mathrm{eff}} \frac{F_{\text {eff }}}{\sqrt{\left(k_{\mathrm{eff}}-m_{\mathrm{eff}} \omega^{2}\right)^{2}+c^{2} \omega^{2}}} \omega \frac{\sqrt{R^{2}-d^{2}}}{R} \cos \theta .
\end{aligned}
$$

Figure 4 shows the effect of the offset distance of the bimorph and the added mass on $f_{\nu}$ and $f_{m}$, which are tangential components of the velocity and momentum of the bimorph when $\theta=20^{\circ}, R=15 \mathrm{~mm}$, and the applied voltage is $50 \mathrm{~V}$.

As the added mass increases, $f_{\nu}$ decreases and $f_{m}$ increases. $f_{\nu}$ and $f_{m}$ hold maximum values at $d=4 \mathrm{~mm}$ and $d$ $=7 \mathrm{~mm}$, respectively. Here, we can establish a guideline for designing the proposed motor. While the offset distance should be determined in the range between 7 and $4 \mathrm{~mm}$ to maximize the velocity and torque of the bimorph, a compromise of added mass is required since the characteristics of the proposed motor are in conflict with each other, that is, the velocity and momentum.

\section{MOTOR FABRICATION}

Figure 5 shows the basic structure of the fabricated piezoelectric motor. The stator is made from engineering plastic by machining and is glued to four T220-A4-203X bimorphs (Piezo System, Inc). Urethane rubbers are attached to the tip of the bimorphs to control the contact condition between the rotor and stator according to the selected contact area and thickness. This allows the bimorph to transmit momentum to

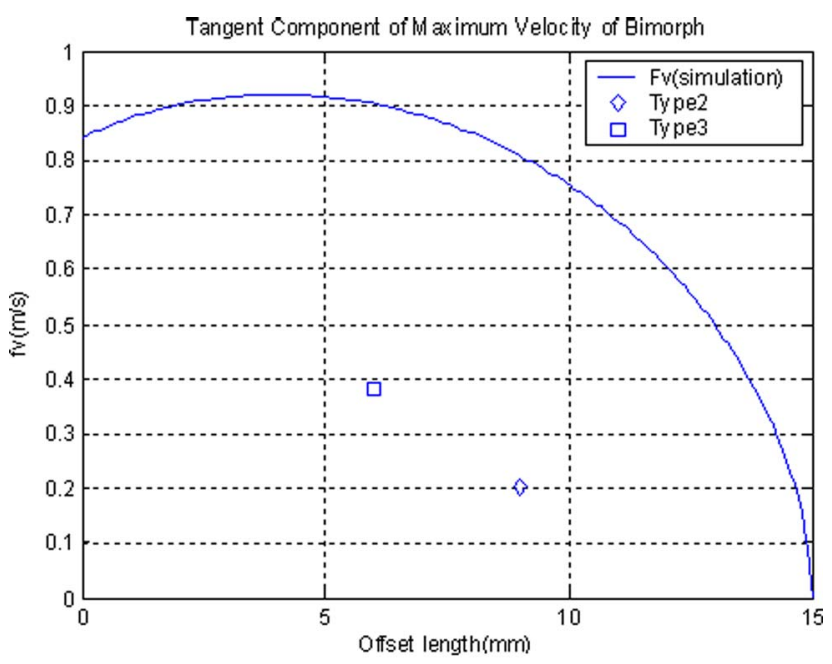

FIG. 7. Comparison between experiments and simulation. 
the rotor effectively. The weight and inertia of the proposed motor are $2.905 \mathrm{~g}$ and $3.6 \mathrm{~g} \mathrm{~cm}^{2}$, respectively. Each urethane rubber has a weight of $0.034 \mathrm{~g}$ and a radial bearing is placed in the center of the stator to stabilize the rotation of the rotor. The preload to the rotor can be adjusted by controlling the displacement of the contracted spring, which is located at the top of the rotor.

\section{EXPERIMENT}

\section{A. Performance of motor}

The experimental setup for the velocity measurement is presented in Fig. 6(a). It consists of a function generator, a voltage amplifier, and a digital tachometer. A sinusoidal signal generated by the function generator is amplified ten times, causing the bimorph to vibrate in resonant frequency. The rotation velocity is measured using a digital tachometer in a noncontact procedure.

The experimental setup for the static torque measurement is presented in Fig. 6(b). Here we measured the tangential force and estimated the static torque.

In order to compare the experiment results with the theoretical guideline, various types of motors were fabricated, varying the offset distance of the bimorph as shown in Table I. Other parameters not listed in Table I are the same as each other and each type of the bimorph operated in its own resonant frequency.

Figure 7 shows a comparison between the guideline and experimental results. Comparing type 2 with type 3 , the velocity characteristic of the fabricated motor is analogous to the performance index $f_{\nu}$, making $f_{\nu}$ reliable. The fabricated motor is operated for more than $1 \mathrm{~h}$ without heat generation or a drastic change in the velocity or torque.

Figure 8 shows the steady-state velocity of the three fabricated motors. Up to $30 \mathrm{~V}$ of operation voltage, the bimorphs could not rotate the rotor because their deflection was too small. The steady-state angular velocity of the proposed motor is linearly proportional to the applied voltage in the range of $30-80 \mathrm{~V}$. It is thus expected that the motor will be

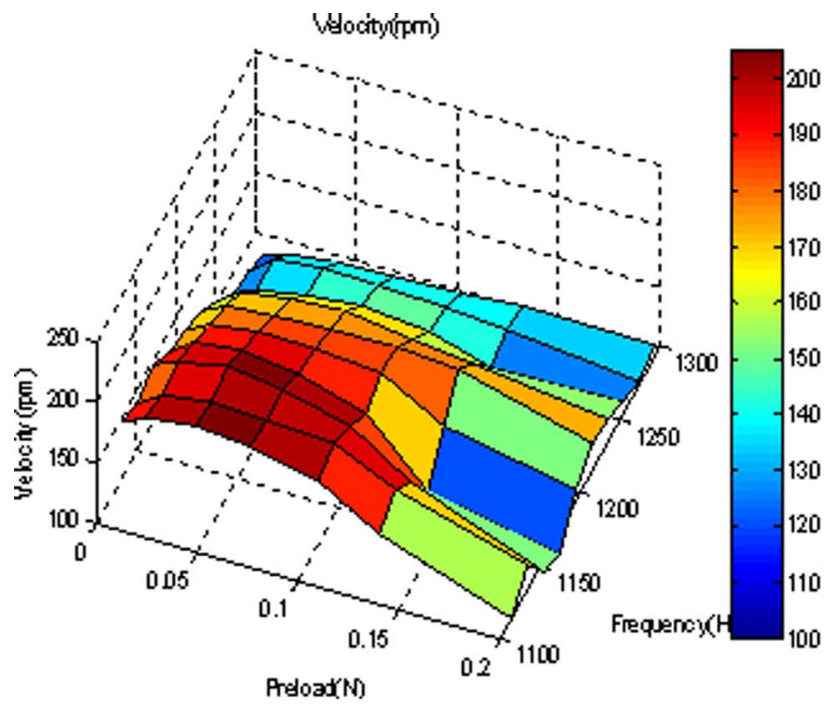

(a)

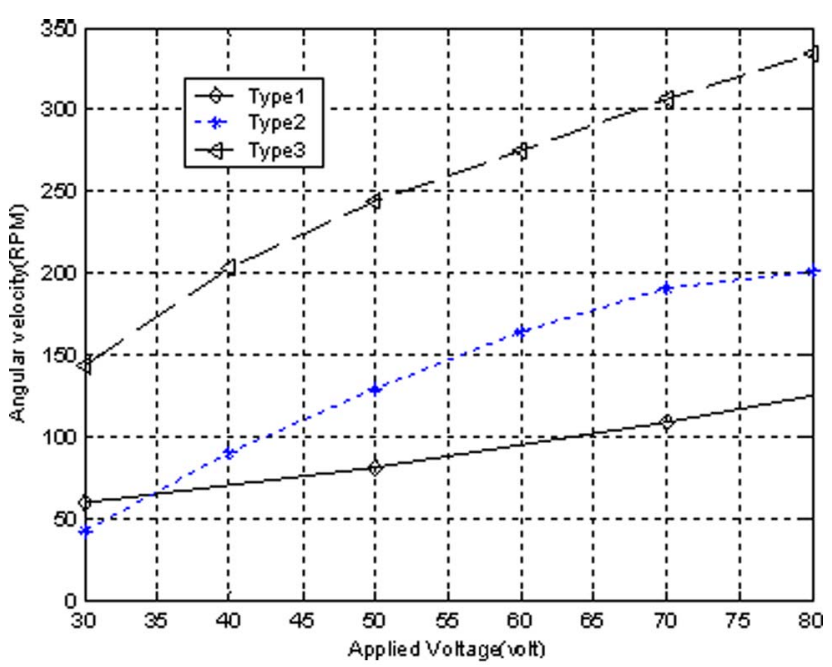

FIG. 8. Angular velocity of piezoelectric motors.

easily controllable. However, the velocity and torque of the proposed motor were significantly dependent upon the surface conditions of the rotor, the pressing force loaded to the rotor, and the operation method.

Accordingly, some attempts have been made to determine the parameters having dominant effects on the velocity and torque of the proposed piezoelectric motor. The operation frequency, operation voltage, preload, and operation method of the four bimorphs were considered in this regard. Additional experiments were performed to determine the effects of these parameters on the velocity and torque of the proposed motor.

First, we measured the steady-state velocity and static torque of the proposed motor with varying preload and operation frequency. The experiment results are presented in Fig. 9. Figure 9(a) shows the steady-state velocity of the piezoelectric motor when the applied voltage is $100 \mathrm{~V}$. The maximum velocity, $200 \mathrm{rpm}$, is obtained at a resonant frequency of $1140 \mathrm{~Hz}$ and a preload of $0.05 \mathrm{~N}$. As the difference between the operation frequency and the resonant fre-

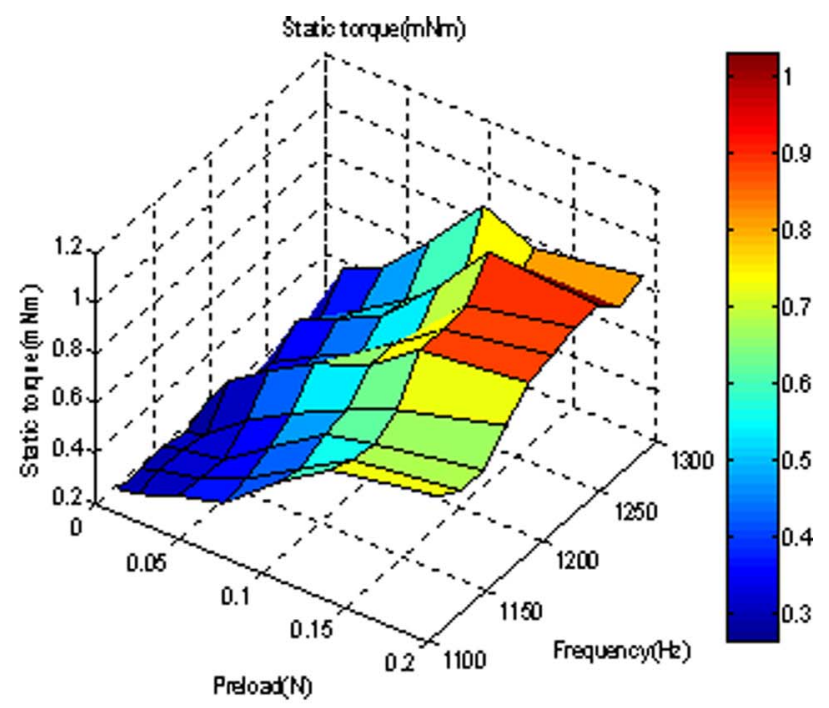

(b)

FIG. 9. Velocity and torque vs preload and frequency. (a) Velocity vs preload and frequency. (b) Torque vs preload and frequency. 


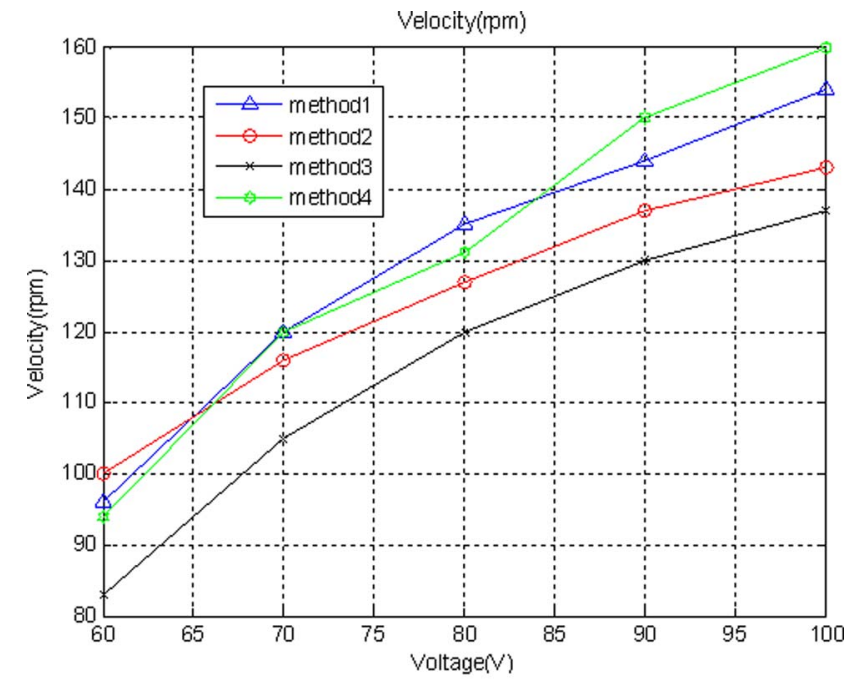

FIG. 10. Velocity of the motor vs voltage and operation method.

quency increases, the velocity decreases. In addition, the velocity decreases gradually with higher preload. Figure 9(b) shows the static torque of the piezoelectric motor with the same conditions as employed in the velocity measurement. While there is a specific value of the preload that maximizes the velocity of the motor, the static torque is proportional to a preload under $0.14 \mathrm{~N}$, due to energy transfer via friction from the bimorph to the rotor. As the preload increases, the contact friction is increased and the slipping energy is reduced between the bimorph and the rotor. However, the limitation of the preload is about $0.2 \mathrm{~N}$. Furthermore, it is not expected that the motor will perform above $0.2 \mathrm{~N}$. The maximum torque is $1.1 \mathrm{mNm}$ at an operation frequency of $1280 \mathrm{~Hz}$ and a preload of $0.14 \mathrm{~N}$. Unlike the findings for the velocity measurement, the output torque is not sensitive to a change of frequency.

\section{B. Characteristic experiments of driving method}

In order to obtain an appropriate operation method in terms of both the static torque and the steady-state velocity, it

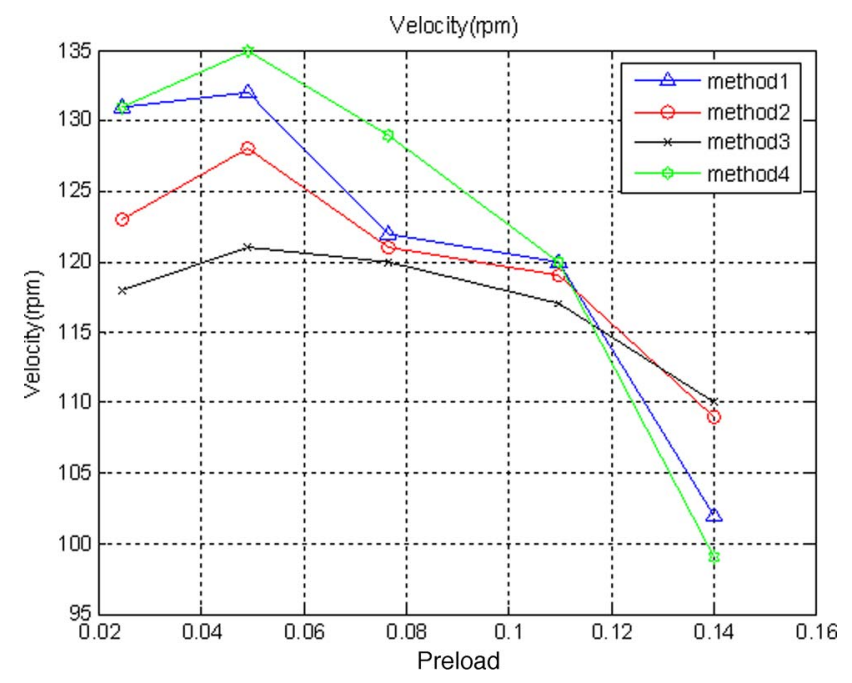

FIG. 11. Static torque of the motor vs voltage and operation method.

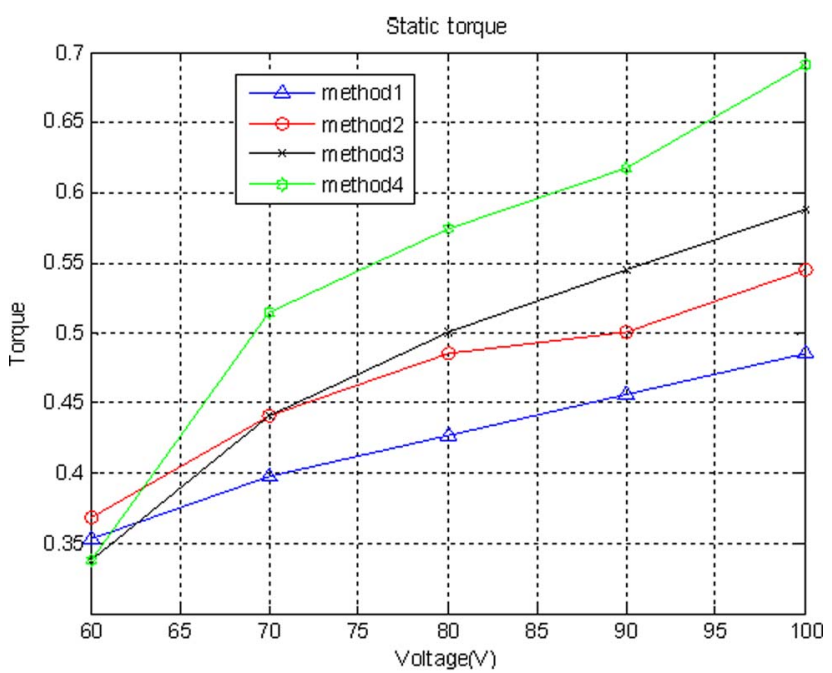

FIG. 12. Velocity of the motor vs preload and operation method.

is necessary to apply various operation methods to the bimorph. The operation methods of the bimorphs are defined as shown in Eq. (19) so as to observe the effects of the phase shift of the bimorphs on the motor characteristics. Each bimorph was operated with independent voltage signal.

$$
\begin{aligned}
& \text { method 1: } \nu_{1}=\nu_{0} \sin (w t), \quad \nu_{2}=\nu_{0} \sin (w t), \quad \nu_{3} \\
& =\nu_{0} \sin (w t), \quad \nu_{4}=\nu_{0} \sin (w t), \\
& \text { method 2: } \nu_{1}=\nu_{0} \sin (w t), \quad \nu_{2}=\nu_{0} \sin \left(w t+\frac{\pi}{2}\right), \quad \nu_{3} \\
& =\nu_{0} \sin (w t), \quad \nu_{4}=\nu_{0} \sin \left(w t+\frac{\pi}{2}\right),
\end{aligned}
$$

method 3: $\nu_{1}=\nu_{0} \sin (w t), \quad \nu_{2}=\nu_{0} \sin (w t+\pi), \quad \nu_{3}$

$$
=\nu_{0} \sin (w t), \quad \nu_{4}=\nu_{0} \sin (w t+\pi)
$$

$$
\begin{aligned}
\operatorname{method} 4: \nu_{1} & =\nu_{0} \sin (w t), \quad \nu_{2}=\nu_{0} \sin \left(w t+\frac{\pi}{2}\right), \quad \nu_{3} \\
& =\nu_{0} \sin (w t+\pi), \quad \nu_{4}=\nu_{0} \sin \left(w t+\frac{3 \pi}{2}\right),
\end{aligned}
$$

At an operation frequency of $1220 \mathrm{~Hz}$, the velocity and static torque are linearly proportional to the voltage for all operation methods, as shown in Figs. 10 and 11, while the maximum velocity and maximum torque are obtained with the fourth operation method, wherein voltage was applied sequentially with a $90^{\circ}$ phase shift.

The velocity of the rotor is highest with a preload of $0.05 \mathrm{~N}$, as shown in Fig. 12, for each operation method. And maximum static torque is also obtained in operation method 4 with increasing preload as shown in Fig. 13. Examining the effects of the operation method under voltage and preload, operation method 4 is found to be the most suitable to drive the proposed motor, as it yields the maximum velocity and static torque. 


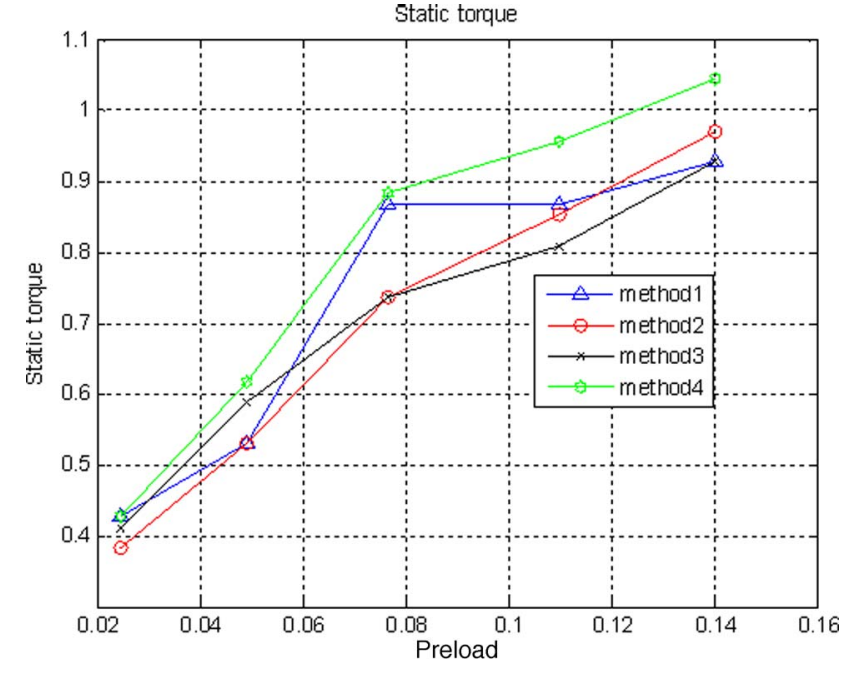

FIG. 13. Static torque of the motor vs preload and operation method.

\section{DISCUSSION}

This article presents the development of a new piezoelectric motor that could overcome excessive heat generation and wear induced by friction. A performance evaluation of the proposed motor in relation to frequency, voltage, preload, and operation method is also presented. The rotor is basically disk shaped with an added shaft. AC voltage is applied to four bimorphs attached to the inclined side of the stator and they vibrate in resonant frequency. In order to maximize the performance of the proposed motor, the authors established design guidelines with the assumption that the angular velocity is proportional to the tangential velocity of the bimorph's end and the static torque is proportional to the tangential component of the momentum generated by the bimorph. The reliability of $f_{\nu}$ is partially verified by comparison between the guideline and velocity of each motor fabricated with a different offset distance. The fabricated motor did not show heat generation during $1 \mathrm{~h}$ of testing, and the effects of frequency, preload, and operation method on the motor were experimentally determined.

Future work will focus on miniaturizing the motor upon the consideration that high blocked force is obtained with decreasing length of the bimorph, and optimizing the shape of the rotor based on the design guidelines.

${ }^{1}$ K. Uchino, Piezoelectric Actuator and Ultrasonic Motor (Kluwer, Dordrecht, 1997), p. 265.

${ }^{2}$ M. A. Dubois and P. Muralt, IEEE Trans. Ultrason. Eng. 45, 1169 (1998).

${ }^{3}$ H. V. Barth, IBM Tech. Discl. Bull. 16, (1973).

${ }^{4}$ T. Shashida, Mech. Automation Japan 15, 31 (1983).

${ }^{5}$ T. Kasuga, T. Satoh, N. Tsukada, T. Yamazaki, F. Ogawa, M. Suzuki, I. Horikoshi, and T. Itoh, J. Soc. Precis. Eng. 57, 63 (1991).

${ }^{6}$ A. Iino, K. Suzuki, M. Kasuga, M. Suzuki, and T. Yamanaka, Ultrasonics 38, 54 (2000).

${ }^{7}$ J. G. Smits and A. Ballato, J. Microelectromech. Syst. 3, 105 (1994).

${ }^{8}$ L. Meirovitch, Principles and Techniques of Vibrations (Prentice-Hall, Englewood Cliffs, NJ, 1997). 